\title{
Pancreatic Cancer: Overview of Recommendations from the 2018 National Institute of Clinical Excellence (NICE) Guidelines on Providing pain relief to Patients with Endoscopic Therapies
}

\author{
AI Koulouris ${ }^{1,2 *}$, KJ Roberts ${ }^{3}$ and AR Hart ${ }^{1,2}$ \\ ${ }^{1}$ Norwich Medical School, University of East Anglia, United Kingdom \\ ${ }^{2}$ Norfolk and Norwich University Hospital, United Kingdom \\ ${ }^{3}$ University of Birmingham, United Kingdom
}

*Corresponding author: Andreas I Koulouris, Bob Champion Research Centre, Norwich, United Kingdom.

Received Date: June 14, 2019

Published Date: June 18, 2019

\section{Mini Review}

EUS-CPN is an outpatient endoscopic procedure and is recommended within current NICE as part of the standard care for relieving abdominal pain in patients with pancreatic cancer, when pain is intractable, or morphine use is escalating. . A meta-analysis of case series report that EUS-CPN can significantly reduce pain scores $(\mathrm{p}<0.00001)$. In a clinical trial, compared to patients treated with morphine alone, those receiving EUS-CPN had $60.7 \%$ less pain $(\mathrm{P}=0.01)$ at 3 months. Diarrhea, postural hypotension and exacerbation of pain occurs in $9-30 \%$ of patients, which resolve spontaneously. Spinal stroke has been reported twice in case reports. Overall, EUS-CPN reduces pain and morphine consumption, and should be available routinely, although further research is needed to optimize timing and patient selection. In their guidelines from 2018 on the management of patients with pancreatic cancer, NICE recommends use of endoscopic ultrasound guided coeliac plexus neurolysis (EUS-CPN) to promote relief of abdominal pain when the pain: i) is intractable, ii) is refractory to escalating doses of opioids or iii) there are adverse effects from opioids negatively affecting patient's quality of life [1]. This paper gives an overview of EUS-CPN use, which is relevant to many health professionals who manage patients with pancreatic cancer. Currently, EUS-CPN availability may be limited due to the relative lack of doctors trained in this procedure. We review its application, efficacy and safety which clinicians need to be appreciate when referring patients for EUS-CPN.
Pancreatic cancer has the worst prognosis of any cancer, and only minimal survival benefits have been achieved in recent decades. Nearly $80 \%$ of patients report abdominal pain, of which $44 \%$ require strong opioid analgesia. Observational work shows that uncontrolled pain is associated with a reduced survival, performance status and tolerance to chemotherapy [24]. Uncontrolled pain may also negatively affect appetite, mood, sleep and quality of life, hence promoting analgesia is essential. EUS-CPN is performed as a day case procedure, under conscious sedation with midazolam and pethidine given at similar doses for a diagnostic colonoscopy. A flexible endoscope, which has an ultrasound probe incorporated in its tip, is inserted through the oral cavity into the stomach. The probe is then placed against the lesser curve of the stomach enabling the endoscopist to visualize the pancreas ultrasonically on a screen. The coeliac trunk (the root of the coeliac artery arising at the abdominal aorta), which is surrounded by the neuronal coeliac plexus, is visualized on the ultrasound screen. The coeliac trunk is the anatomical landmark for localizing the coeliac plexus which cannot be directly visualized. The endoscopist then advances a needle through the stomach wall into the retroperitoneal space laterally to the coeliac trunk and injects local anesthetic (usually $10 \mathrm{ml} 25 \%$ bupivacaine), followed by $10 \mathrm{~mL}$ of dehydrated ( 98 percent) alcohol around the coeliac trunk. Alcohol causes irreversible chemical ablation of the afferent pain fibres originating from the pancreas, which synapse at the 
coeliac ganglia, and disrupts pain signaling pathways to the brain. Typically, the procedure lasts 30 to 45 minutes and a short period of observation is required in the recovery area before discharge.

The efficacy of EUS-CPN was reported in a meta-analysis of 6 case series of 295 patients which documented a mean decrease of 4.26 points $(95 \%$ CI $3.00-5.53, \mathrm{p}<0.00001)$ in the abdominal pain visual analogue score (1-10 scale) after 2 weeks [5]. This benefit was sustained in two-weekly follow-up measurements of pain score over 3 months ( $\mathrm{p}<0.001)$. However, there is only one randomized controlled trial of 98 patients, which compared EUS-CPN versus opioid analgesia, alone in patients with abdominal pain at diagnosis [6]. Participants in the EUS-CPN group reported reduction in pain scores by $18 \%$ ( $95 \% \mathrm{CI}, 0 \%$ to $37 \%$ ) at the first month and $49 \%$ (95\% CI, 38\% to $61 \%$ ) at 3 months. The non-EUS-CPN patients reported increased pain scores of $11 \%$ (95\% CI, 13\% to $49 \%)$ at the first month and $12 \%$ (95\% CI, $-19 \%$ to $36 \%$ ) at 3 months, when patients in the EUS-CPN group had $60.7 \%$ less pain than those in the control arm [difference between the mean percent changes in pain scores was $-60.7 \%, 95 \% \mathrm{CI},-86.6$ to $-25.5, \mathrm{p}=0.01$ )] [6]. There were several trial limitations namely patients with metastases were excluded, cancer stage was unreported and patients developing pain after diagnosis were not included. Furthermore, nearly $83 \%$ of patients were excluded for unreported reasons which questions its generalizability. Another observational study reported cancers directly infiltrating the coeliac plexus responded less well to EUS-CPN, than those not involving this structure [7]. A possible explanation is that the injectate may not reach pain fibres deeper within the cancer. There may thus be a role for prophylactic EUSCPN among patients with pancreatic cancer given the prevalence of this problem, though the efficacy of this is untested. Other variables such as cancer location within the pancreas, tumour size, pain score at baseline and demographics do not predict response to EUS-CPN [7]. Complications of EUS-CPN are relatively uncommon and selflimiting including diarrhea (18\%), postural hypotension (11-30\%) and worsening abdominal pain (9\%), which resolve within a week $[5,6]$. Spinal stroke causing irreversible paraparesis, described in two patients, is a rare and serious consequence, presumably due to vasospasm of a spinal artery branch after injecting alcohol $[8,9]$. There are no reports of any procedure-associated mortality. Future qualitative research should explore patients' perspectives of receiving endoscopic therapies, as most will already have had a diagnostic EUS to obtain cytology.

Currently, opioids are the first line treatment for controlling abdominal pain in patients with pancreatic cancer as it is a treatment with moderate analgesic effect, is non-invasive and easy to administer. However, opioids also have many serious and debilitating side effects, including nausea, sedation, constipation and cough suppression which impair quality of life. Opioids bind to receptors on nerve cell membranes and oppose formation of action potentials transmitted to the brain. As the cancer progresses though, many patient's loose response to opioids despite increasing doses. EUS-CPN appears to prevent this tendency to increase opioid use over time [6]. Daily morphine consumption increased by an average of $54 \mathrm{mg}$ in the first month since diagnosis and $100 \mathrm{mg}$ in the third month for those randomized to standard care analgesia, whilst it increased by $53 \mathrm{mg}$ in the first month in the EUS-CPN group, but remained static at $50 \mathrm{mg}$ thereafter, although the difference was not statistically significant $(\mathrm{P}=0.10)$ [6]. EUS-CPN may inhibit neuroremodeling as the tumour progresses, when increasing amounts of neurotrophic cytokines are secreted, promoting proliferation of pain-sensing neurons containing opioid receptors. Histological examination of surgical pancreatic specimens from patients with abdominal pain prior to pancreatectomy documented up to 14 times the number of nerves fibres per unit area compared to normal tissue [10]. Nociceptive receptors, absent in normal pancreas, are observed in pancreatic cancer [10]. Despite evidence supporting use of EUS-CPN in the management of patients with abdominal pain with pancreatic cancer, the timing of its administration and patient selection need to be further researched. Currently, EUS-CPN is often administered late in the cancer's natural history, when pain is established, and some patients are receiving increasing doses of opioids. At this time, performance status is often poor, and they have little time to live. EUS-CPN tends to be limited to specialist centers though most patients with pancreatic cancer are managed locally. Thus, research to assess the optimal timing of EUS-CPN are needed as well as awareness and availability of this procedure across networks if the NICE recommendations are to be delivered.

\section{Acknowledgement}

None.

\section{Conflict of Interest}

No conflict of interest.

\section{References}

1. National Institute of Clinical Excellence (NICE) (2018) Pancreatic Cancer in Adults: diagnosis and management, United Kingdom.

2. Moningi S, Walker AJ, Hsu CC, Reese JB, Wang JY, et al. (2015) Correlation of Clinical Stage and Performance Status with Quality of Life in patients seen in a pancreas multidisciplinary clinic. J Oncol Pract 11(2): e216-e221.

3. Müller, Friess, Köninger, Martin D, Wente MN, et al. (2008) Factors influencing survival after bypass procedures in patients with advanced pancreatic adenocarcinomas. Am J Surg 195(2): 221-228.

4. Wang, Fogelman, Shi Q, Cathy E , Raza HB, et al. (2012) Patient-reported symptoms from patients with advanced pancreatic cancer undergoing gemcitabine-based chemotherapy. J Clin Oncol 30(4): 370.

5. Nagels W, Pease N, Bekkering G, Cools F, Dobbels P (2013)Celiac Plexus Neurolysis for Abdominal Cancer Pain: A Systematic Review. Pain Med 14(8): 1140-1163.

6. Wyse, Carone, Paquin, Usatii M, Sahai AV (2011) Randomized, doubleblind, controlled trial of early endoscopic ultrasound-guided celiac plexus neurolysis to prevent pain progression in patients with newly diagnosed, painful, inoperable pancreatic cancer. J Clin Oncol 29(26): 3541-3546.

7. Iwata K, Yasuda I, Enyaet M, Mukai T, Nakashima M, et al. (2011) Predictive factors for pain relief after endoscopic ultrasound-guided celiac plexus neurolysis. Dig Endosc. 23(2): 140-145.

8. Mittal, Rabinstein, Wijdicks (2012) Pearls \& Oy-sters: Acute spinal cord infarction following endoscopic ultrasound-guided celiac plexus neurolysis. Neurology 78(9): e57-e59. 
9. Fujii, Clain, Morris, Levy MJ (2012) Anterior spinal cord infarction with permanent paralysis following endoscopic ultrasound celiac plexus neurolysis. Endoscopy 44: 255-256.
10. Ceyhan, Bergmann F, Kadihasanoglu M, Altintas B, Demir IE, et al. (2009) Pancreatic neuropathy and neuropathic pain--a comprehensive pathomorphological study of 546 cases. Gastroenterology 136(1): 177186. 